\title{
HEAT TRANSFER IN MULTI-CONNECTED AND IRREGULAR DOMAINS WITH NON-UNIFORM MESHES
}

\author{
E. C. Romão, \\ J. B. Aparecido ${ }^{b}$, \\ J. B. Campos-Silvab, \\ L. F. M. de Moura ${ }^{a}$ \\ ${ }^{a}$ Universidade Estadual de Campinas \\ Faculdade de Engenharia Mecânica \\ Departamento de Engenharia Térmica e \\ Fluidos \\ Rua Mendeleyev, 200 \\ Cidade Universitária "Zeferino Vaz" \\ Distrito de Barão Geraldo \\ CEP:13083-860 Campinas SP Brasil \\ estaner23@yahoo.com.br \\ buniversidade Estadual Paulista \\ Departamento de Engenharia Mecânica \\ Av. Brasil Centro, 56 \\ CEP - 15385-000 - Ilha Solteira SP Brasil
}

\section{ABSTRACT}

In this work is presented a numerical solution for temperature profile in two-dimensional diffusion inside irregular multi-connected geometry. The special discretization has been done by two variants of the finite Element Method: Galerkin Finite Element Method (GFEM) and Least Squares Finite Element Method (LSFEM). Three applications are presented. The first for a regular double connected domain; the second for a regular multi-connected domain and the third application for an irregular multi-connected domain. In all applications are considered Dirichlet boundary conditions. The results obtained in the present work are compared with results from Ansys ${ }^{\circledR}$ simulations. The results of each method are presented and discussed and the characteristics and advantages of the methods are also discussed.

Keywords: Finite Element Method, Least Squares, Galerkin, Diffusion, Multi-connected domains

\section{NOMENCLATURE}

$k \quad$ thermal conductivity

$N_{j} \quad$ interpolation function

$N_{\text {node }}$ number of nodes in each finite element

$q_{x} \quad$ heat flux along the $\mathrm{x}$-axis

$q_{y} \quad$ heat flux along the $y$-axis

$\hat{q}_{x}^{e} \quad$ heat flux approximation along $\mathrm{x}$-axis

$\hat{q}_{y}^{e} \quad$ heat flux approximation along t-axis

$u$ temperature

$\hat{u}_{j}^{e} \quad$ temperature approximation in the finite element

$v \quad$ weight function

$v_{i}^{e} \quad$ weight function in the element

\section{Greek symbols}

$\Omega \quad$ two-dimensional domain

$\Omega^{e} \quad$ two-dimensional domain in the element

$\Gamma \quad$ contour of a domain

$\Gamma^{e} \quad$ contour of an element

\section{Subscripts}

node identification of a node

\section{INTRODUCTION}

From 1950 decade, some researchers among them Turner et al. (1956), Clough (1960) and Argyris (1963) began the using of the Galerkin Finite Element Method for the solution of diffusive problems in simple-connected domains obtaining very good results. In Feng (2001), GFEM was applied to solve nonlinear electro-hydrodynamic problems with the diffusive term more strong than the convective term. In Howle (1996), a study of the computational efficiency of the two numerical methods based on the Finite Difference/Galerkin Technique: Reduced Galerkin and Pseudo-Spectral was done in the solution of the steady state convection problem of Rayleigh-Bénard. The author, after presenting the formulation of both methods, shows a numerical test that demonstrates graphically that the Pseudo-Spectral Method use a larger number of iterations than the Reduced Galerkin Method for the convergence of the solution. However, most of the fluid mechanics and heat transfer problems are in multi-connected domains. In last decades, several authors presented works with applications of the Finite Element Method in its variations, for example, Camprub et al. (2000) and Romão et al. (2008). In the last, an error analysis has been done, comparing solutions of the GFEM and LSFEM with analytical solutions for heat conduction problems from Arpaci (1966). Specially, the LSFEM yields to very good 
results. In Romão (2008), is presented an application of LSFEM for numerical solution of diffusive phenomenon in a two-dimensional multi-connected domain. The main characteristics of the LSFEM is to give an algebraic system that in matrix form is always symmetrical and positive defined, independently of the differential operator in the governing equations. GFEM yields to symmetrical matrix in the case of self-adjoint operator. These interesting characteristics of LSFEM can be explored in solution methods for the matricial system. The work has been divided after this Introduction in the following items: Discretization, Numerical Applications, Conclusions, Acknowledgments and References.

\section{DISCRETIZATION}

Firstly, will be presented the discretization of the governing equation for diffusive problems by both GFEM and LSFEM. The governing equation can be written in the form

$$
\begin{gathered}
\frac{\partial}{\partial x}\left(k \frac{\partial u}{\partial x}\right)+\frac{\partial}{\partial y}\left(k \frac{\partial u}{\partial y}\right)+B u+f=0, \\
x \in[a, b]
\end{gathered}
$$

where the thermal conductivity is assumed to be constant, $k=$ constant $\neq 0, u=u(x, y), B=B(x, y)$ e $f=f(x, y)$ with $x, y \in \mathfrak{R}$ and the boundary conditions are of the first type or Dirichlet boundary conditions.

At the end, three applications are presented comparing results of GFEM and LSFEM with results from Ansys ${ }^{\circledR}$ software.

Now, the spacial discretization of Eq. (1) for the two variants of the FEM: GFEM and LSFEM will be presented.

\subsection{Galerkin Finite Element Method- GFEM}

For the spacial discretization, initially, the function $\mathrm{u}(\mathrm{x}, \mathrm{y})$ described in Eq. (1) is approximated by a function $\hat{u}$, as follows

$$
u \approx \hat{u}^{e}=\sum_{j=1}^{N_{\text {nodes }}} N_{j} \hat{u}_{j}^{e}
$$

where $N_{\text {nodes }}$ is the number of nodes in each element, $N_{j}$ are the interpolation functions and $\hat{u}_{j}^{e}$ are approximations of $u$ in the element. With the approximation defined in Eq. (2) form Eq. (1) can be defined a residual in the

$$
R=\frac{\partial}{\partial x}\left(k \frac{\partial \hat{u}}{\partial x}\right)+\frac{\partial}{\partial y}\left(k \frac{\partial \hat{u}}{\partial y}\right)+B \hat{u}+f
$$

Then, in the application of GFEM, a variational formulation of problem (1) is defined as follows. Find $\hat{u}^{e} \in V^{e}, V^{e} \in C^{2}(\Omega)$, such as

$$
\int_{\Omega^{e}} R v_{i}^{e} d \Omega=0, \forall v_{i}^{e} \in V^{e}, i=1,2, \ldots, N_{\text {nós }} .
$$

where $\Omega \subset \mathfrak{R}^{2}$ is a limited closed domain.

The Eq. (4) will be valid for whichever $v_{i}^{e}=N_{i}$, $i=1,2, \ldots, N_{\text {nodes }}$, so, in GFEM the weight function is assumed to be the proper interpolation function. From Eq. (4) after several manipulations it's obtained the linear system

$$
[K]\left\{\hat{u}^{e}\right\}=\{F\}
$$

in which

$$
K_{i j}=\int_{\Omega^{e}}\left[-k \frac{\partial N_{i}}{\partial x} \frac{\partial N_{j}}{\partial x}-k \frac{\partial N_{i}}{\partial y} \frac{\partial N_{j}}{\partial y}+B N_{i} N_{j}\right] d \Omega
$$

$$
\begin{aligned}
F_{j}= & -\oint_{\Gamma^{e}} N_{j} k \frac{\partial u}{\partial x} \cos \theta d \Gamma- \\
& \oint_{\Gamma^{e}} N_{j} k \frac{\partial u}{\partial y} \operatorname{sen} \theta d \Gamma-\int_{\Omega^{e}} f N_{j} d \Omega
\end{aligned}
$$

with $i=1,2, \ldots, N_{\text {nodes }}$. For more details, see Romão (2004).

\subsection{Least Squares Finite Element Method - LSFEM}

In the application of LSFEM are introduced auxiliary variables in order to reduce the second order partial equation (1) to a system of first order partial differential equations. In this case of two-dimensional diffusion are introduced the fluxes in both directions:

$$
q_{x}=-k \frac{\partial u}{\partial x}, \quad q_{y}=-k \frac{\partial u}{\partial y}
$$

It's more convenient for algebraic manipulation to discretize first order partial differential equations when using LSFEM than second order equation. By combining the Eqs. (8a-b) and Eq. (1), the following system is obtained

$$
-\frac{\partial q_{x}}{\partial x}-\frac{\partial q_{y}}{\partial y}+B u+f=0,
$$




$$
\begin{gathered}
q_{x}+k \frac{\partial u}{\partial x}=0, \\
q_{y}+k \frac{\partial u}{\partial y}=0,
\end{gathered}
$$

Similar to the GFEM, the variables $u, q_{x}$ e $q_{y}$, are approximated by the $\hat{u}, \hat{q}_{x}$ e $\hat{q}_{y}$, in the following way

$$
\begin{aligned}
& u \approx \hat{u}^{e}=\sum_{j=1}^{N_{\text {nós }}} N_{j} \hat{u}_{j}^{e} \\
& q_{x}=\hat{q}_{x}^{e}=\sum_{j=1}^{N_{\text {nós }}} N_{j} \hat{q}_{x j}^{e} \\
& q_{y}=\hat{q}_{y}^{e}=\sum_{j=1}^{N_{\text {nós }}} N_{j} \hat{q}_{y}^{e}
\end{aligned}
$$

and since $\hat{u}, \hat{q}_{x}$ and $\hat{q}_{y}$ are approximations to $u$, $q_{x}$ e $q_{y}$, then, from Eqs. (9)-(11) the residuals can be defined

$$
\begin{aligned}
& R_{1}=-\frac{\partial \hat{q}_{x}}{\partial x}-\frac{\partial \hat{q}_{y}}{\partial y}+B \hat{u}+f \\
& R_{2}=\hat{q}_{x}+k \frac{\partial \hat{u}}{\partial x} \\
& R_{3}=\hat{q}_{y}+k \frac{\partial \hat{u}}{\partial y}
\end{aligned}
$$

In the LSFEM should be found $\hat{u}^{e} \in V^{e}$ in such way to minimize the functional (Jiang, 1998)

$$
\begin{aligned}
& I\left(R_{1}, R_{2}, R_{3}\right)=\int_{\Omega^{e}} R_{1}^{2}(x, y) d \Omega+ \\
& \quad \int_{\Omega^{e}} R_{2}^{2}(x, y) d \Omega+\int_{\Omega^{e}} R_{3}^{2}(x, y) d \Omega
\end{aligned}
$$

The condition to minimize a functional is that its first variation be null. In this way, from Eq. (18) results

$$
\begin{array}{r}
\int_{\Omega^{e}}\left(\delta R_{1}\right) R_{1} d \Omega+\int_{\Omega^{e}}\left(\delta R_{2}\right) R_{2} d \Omega+ \\
\int_{\Omega^{e}}\left(\delta R_{3}\right) R_{3} d \Omega=0
\end{array}
$$

By substitution of the residuals and the first variation of them in Eq. (19) the following linear is obtained

$$
\begin{aligned}
& {\left[\begin{array}{ccc}
A & B & C \\
B^{T} & D & E \\
C^{T} & E^{T} & G
\end{array}\right]\left\{\begin{array}{l}
\left\{\begin{array}{l}
\hat{u}_{i}^{e} \\
\hat{q}_{x_{i}}^{e} \\
\hat{q}_{y_{i}}^{e}
\end{array}\right\} \\
i=1,2, \ldots, \text { Nnodes },
\end{array}\right.} \\
& i=\left\{\begin{array}{l}
F_{1 i} \\
F_{2 i} \\
F_{3 i}
\end{array}\right\},
\end{aligned}
$$

in which the elements of the matrices are defined as follows

$A_{i j}=\int_{\Omega^{e}}\left\{B^{2} N_{i} N_{j}+k^{2} \frac{\partial N_{i}}{\partial x} \frac{\partial N_{j}}{\partial x}+k^{2} \frac{\partial N_{i}}{\partial y} \frac{\partial N_{j}}{\partial y}\right\} d \Omega$,

$B_{i j}=\int_{\Omega^{e}}\left\{-B N_{i} \frac{\partial N_{j}}{\partial x}+k \frac{\partial N_{i}}{\partial x} N_{j}\right\} d \Omega$,

$C_{i j}=\int_{\Omega^{e}}\left\{-B N_{i} \frac{\partial N_{j}}{\partial y}+k \frac{\partial N_{i}}{\partial y} N_{j}\right\} d \Omega$,

$D_{i j}=\int_{\Omega^{e}}\left[\frac{\partial N_{i}}{\partial x} \frac{\partial N_{j}}{\partial x}+N_{i} N_{j}\right] d \Omega$,

$E_{i j}=\int_{\Omega^{e}} \frac{\partial N_{i}}{\partial x} \frac{\partial N_{j}}{\partial y} d \Omega$

$G_{i j}=\int_{\Omega^{e}}\left[\frac{\partial N_{i}}{\partial y} \frac{\partial N_{j}}{\partial y}+N_{i} N_{j}\right] d \Omega$,

$F_{1 i}=\int_{\Omega^{e}}\left[B N_{i}\right] \times(-f) d \Omega$,

$F_{2 i}=\int_{\Omega^{e}} \frac{\partial N_{i}}{\partial x} f d \Omega$

$F_{3 i}=\int_{\Omega^{e}} \frac{\partial N_{i}}{\partial y} f d \Omega$,

with $i, j=1, \ldots$, Nnodes .

\section{NUMERICAL APPLICATIONS}

The integrations in Eqs. (6) and (7) and (21)-(29) are done by application of the Gauss quadrature (Reddy, 1993) by using parametrical elements mapped in local coordinates $\xi$ and $\eta(-1 \leq \xi, \eta \leq 1)$. This procedure can be also found in Romão (2004). The interpolation function for triangular and quadrilateral elements are found in Dhatt and Thouzot. (1984).

In that follows, three applications are presented. First, a diffusive problem in a double connected domain is analyzed. Second, a multi-connected 
domain is considered and the third application is in an irregular multi-connected domain. The objective is to analyzing the performance of GFEM and LSFEM in three meshes previously defined.

\section{Application 1: Pure Diffusion in a double connected geometry.}

In this application a pure diffusion problem in a double connected geometry is considered. Double connected means an external and an internal boundary. In this case, $k=1$ and $B=f=0$ in Eq. (1).

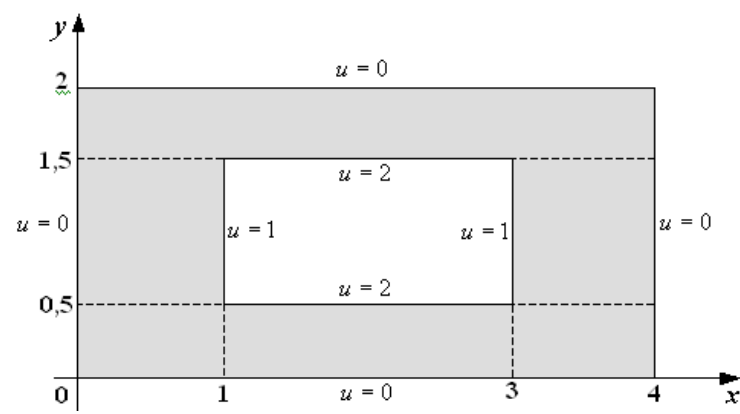

Figure 1. Diffusion in a double connected geometry with boundary conditions of first type.

The non uniform irregular mesh for this case is illustrated by Figure 2. There are 726 quadrilateral elements with eight nodes pert element. The isotherms from Ansys and by GFEM and LSFEM, present work, are shown in Figures 3, 4 and 5 respectively.

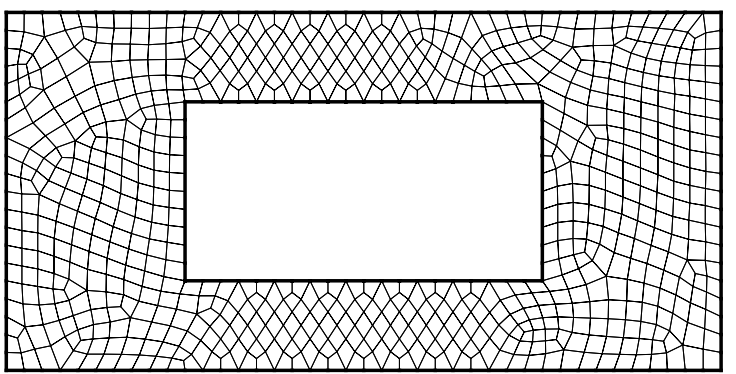

Figura 2. Mesh with 726 quadrlateral elements.

A malha utilizada para solução deste problema contém 726 elementos quadrilaterais com oito nós cada. Para este caso não foi utilizado elementos contendo a mesma área e formato, Fig. 2.

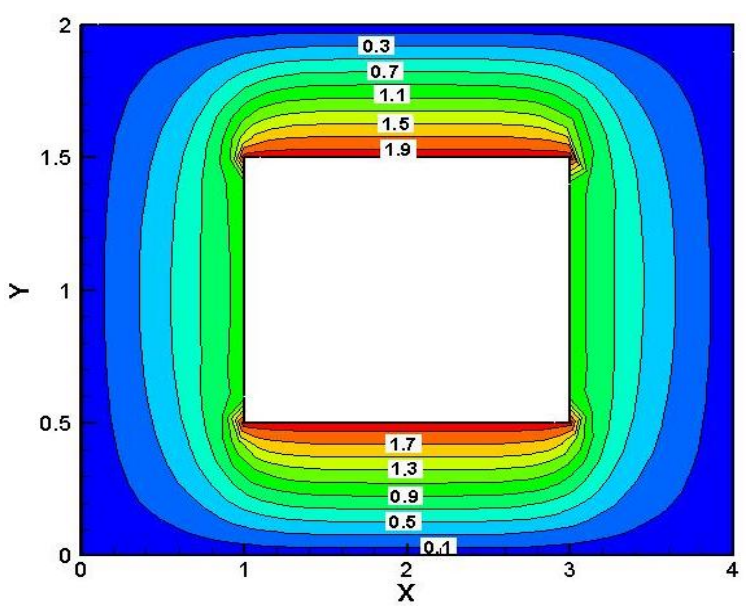

Figure 3. Ansys solution for $u(x, y)$.

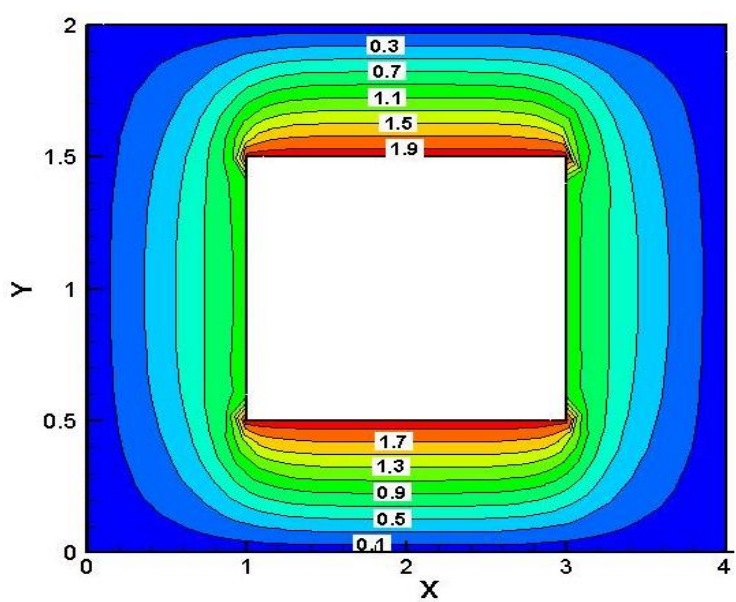

Figure 4. GFEM solution for $u(x, y)$ from the present work.

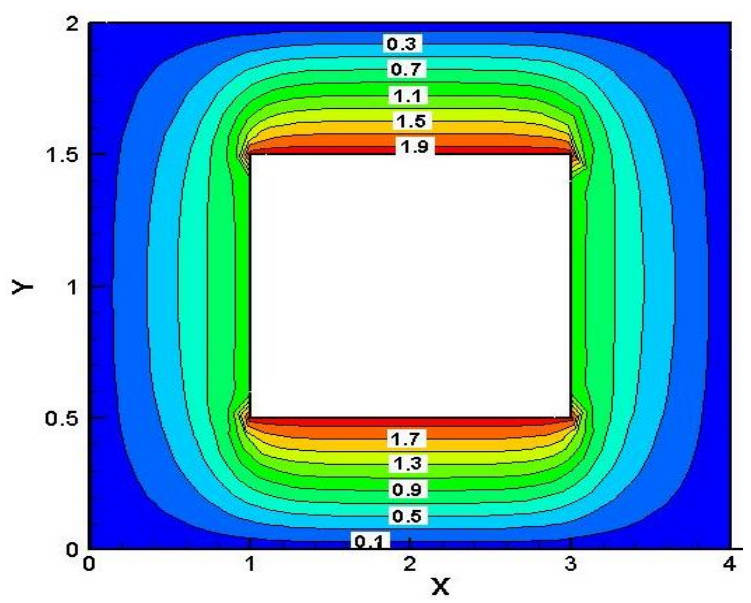

Figure 5. LSFEM solution for $u(x, y)$ from the present work. 
Figures 3, 4 and 5 show that the solutions are in good agreement. Numerical results are presented in Table 1. Our code produced results in very good agreement with simulations from Ansys software.

Table 1. Numerical results for $u(x, y)$ in four points of Application 1.

\begin{tabular}{|c|c|c|c|}
\hline $\mathbf{x}$ & $\mathbf{y}$ & Galerkin & LS \\
\hline 0,96578 & 0,13576 & 0,37513 & 0,37607 \\
\hline 3,01736 & 0,16592 & 0,47215 & 0,47324 \\
\hline 2,00000 & 0,17600 & 0,70301 & 0,70303 \\
\hline 2,05000 & 1,60224 & 1,59032 & 1,59034 \\
\hline
\end{tabular}

\section{Application 2: Pure diffusion in multi-connected domain}

In this application a pure diffusion problem in a multi-connected geometry is considered. Multi-connected means there an external and more than one internal boundary. In this case, $k=0$ and $B=f=0$ in Eq. (1).

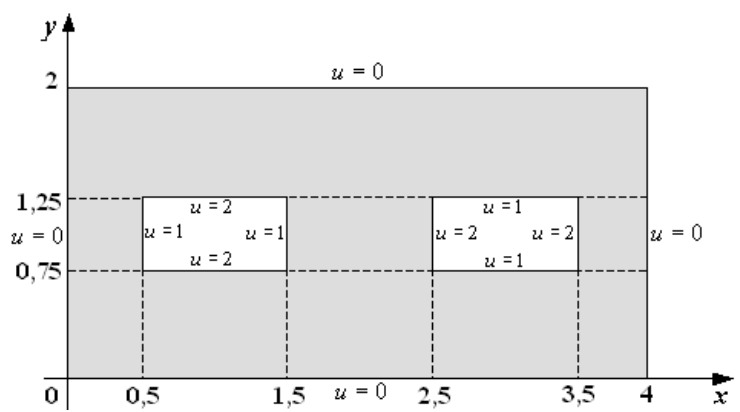

Figure 6. Diffusion in a multi-connected geometry with boundary conditions of first type.

In this application, the main objective is to consider a multi-connected domain. Now the domain is discretized by quadratic six-noded triangular elements. There 966 elements in the mesh illustrated in Figure 7. The results, from the two variants: GFEM and LSFEM, are compared with simulations by Ansys software. The results for isotherms are shown in Figures 8 to 10.

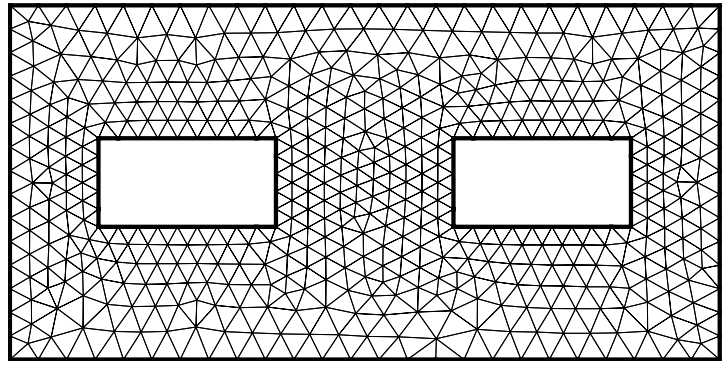

Figure 7. Mesh with 966 quadratic triangular elements.

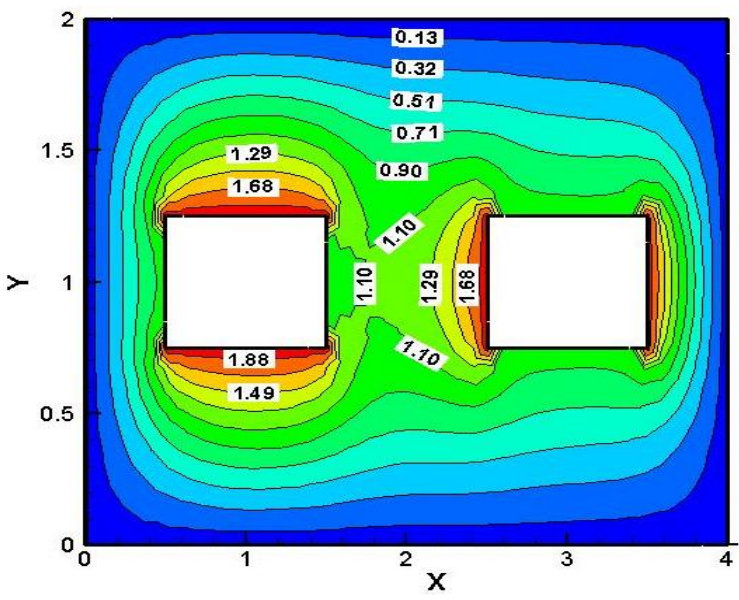

Figure 8. Ansys solution for $u(x, y)$.

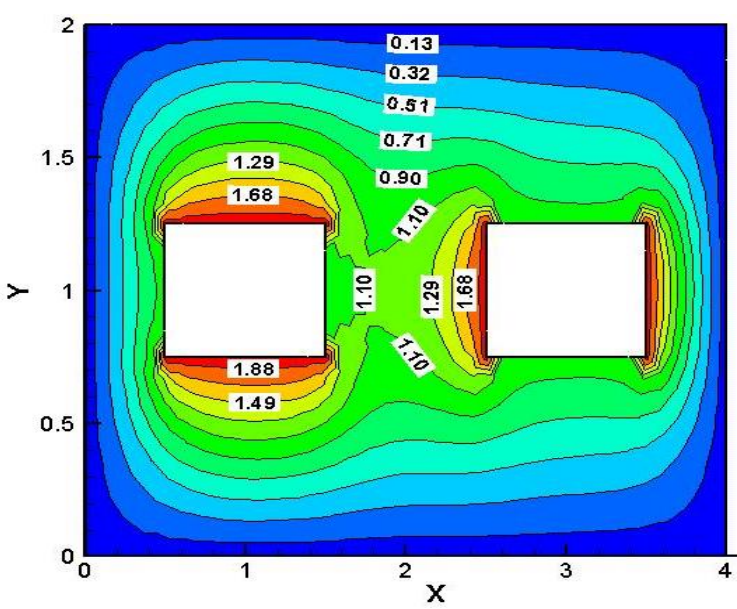

Figure 9. GFEM solution for $u(x, y)$ from the present work.

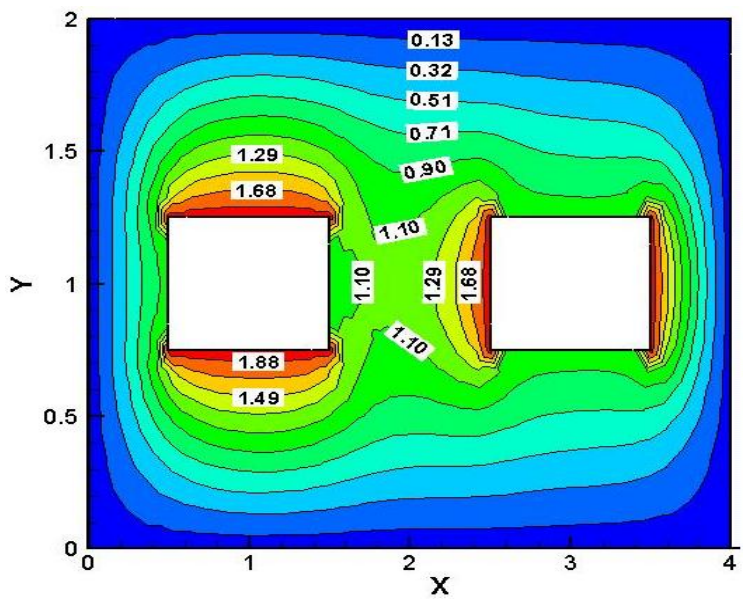

Figure 10. LSFEM solution for $u(x, y)$ from the present work.

Qualitatively, Figures 8 to 10 show similarity of our results with Ansys results. Numerical results 
from simulations for this application are presented in Table 2. Notice the very good agreement between the results from our simulations.

Table 2. Numerical results for $u(x, y)$ in some points of Application 2.

\begin{tabular}{|l|l|l|l|}
\hline $\mathbf{x}$ & $\mathbf{y}$ & Galerkin & LS \\
\hline 1,88165 & 0,63880 & 0,96942 & 0,97379 \\
\hline 0,12826 & 0,99822 & 0,28166 & 0,28213 \\
\hline 1,77624 & 1,20128 & 1,09015 & 1,09414 \\
\hline 3,59203 & 1,10068 & 1,52621 & 1,52680 \\
\hline 2,60976 & 1,92584 & 0,11416 & 0,11198 \\
\hline 1,08364 & 1,30007 & 1,84865 & 1,84888 \\
\hline
\end{tabular}

Application 3: Pure diffusion with thermal generation in a irregular multi-connected geometry.

In this application a pure diffusion problem with thermal generation in an irregular multi-connected geometry is considered. Now, the governing equation (Eq. (1)) includes a source term, $f=7, k=1$ and $B=0$.

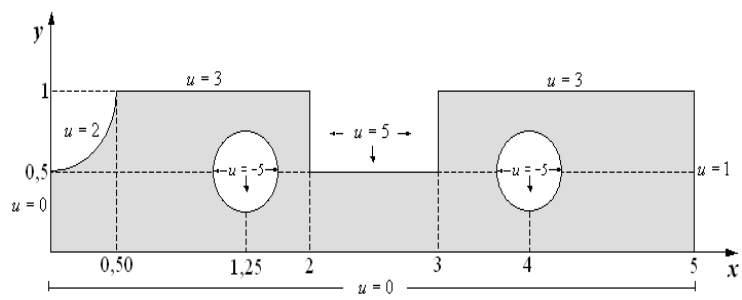

Figure 11. Diffusion in an irregular multi-connected geometry with boundary condition of first type.

For this case, a mesh of 451 quadratic eight-noded elements has been created. It's illustrated by Figure 12. The isotherms for this application are shown in Figures 13 to 15. Figure 13 is for Ansys solution and Figures 14 and 15 are from our code.

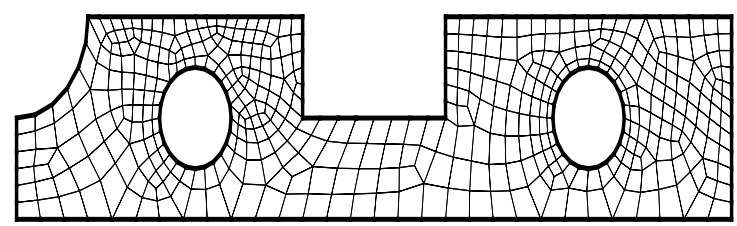

Figure 12. Mesh with 451 quadratic eight (serendipity) elements.

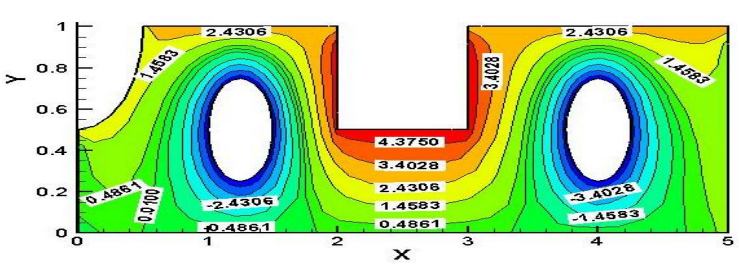

Figure 13. Ansys solution for $u(x, y)$.

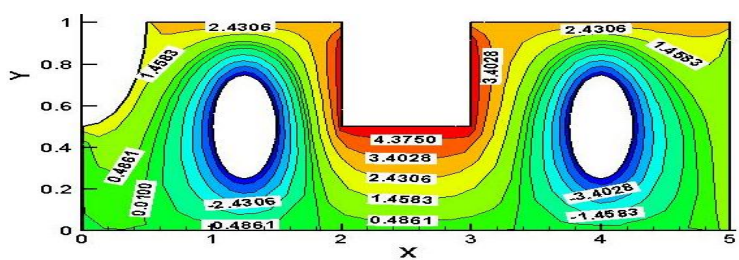

Figure 14. GFEM solution for $u(x, y)$ from the present work.

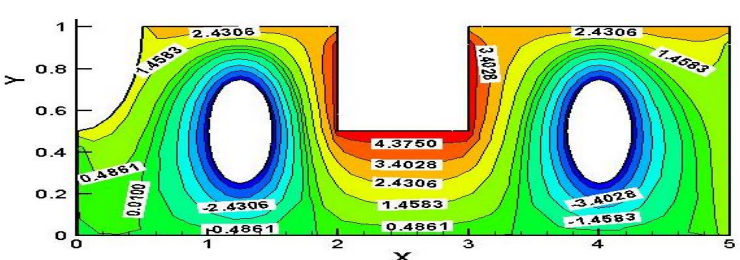

Figure 15. LSFEM solution for $u(x, y)$ from the present work.

Even, for the mesh with only 451 elements there a good agreement between our results and Ansys results. Some numerical results are also presented in Table 3.

Table 3. Numerical results for $u(x, y)$ in some points of Application 3.

\begin{tabular}{|l|l|c|l|}
\hline $\mathbf{x}$ & $\mathbf{y}$ & Galerkin & LS \\
\hline 1,57978 & 0,71147 & $-1,19681$ & $-1,19340$ \\
\hline 0,58999 & 0,86319 & 1,19519 & 1,19510 \\
\hline 2,20324 & 0,12281 & 1,05095 & 1,05596 \\
\hline 3,18820 & 0,91010 & 3,16021 & 3,16127 \\
\hline 4,81200 & 0,70282 & 0,97320 & 0,96960 \\
\hline 4,30027 & 0,81078 & $-0,51380$ & $-0,51548$ \\
\hline
\end{tabular}

\section{CONCLUSION}

In this work has been done a comparison of two variants of the finite element method. Galerkin and Least-Squares methods. Both the GFEM and LSFEM are usefulness tools for solve this kind problem. The results of our code are in very good agreement with results from Ansys package. In the LSFEM there are more degrees of freedom per node due to the auxiliary variables and it's more time consuming. However, the heat fluxes are already calculated in LSFEM without needing of any post-processing. For 
pure diffusion the GFEM is the most appropriated method and it's of easier implementation than the LSFEM. The advantage of LSFEM is when it's necessary the calculation of the heat fluxes, because the solution return also these values. The GFEM need a post-processing to calculate the heat fluxes, Romão (2008).

\section{ACKNOWLEDGMENTS}

The present work was supported by the National Council of Scientific Development and Technology $\mathrm{CNPq}$ - Brasil, in form of scholarship for the first author.

\section{REFERENCES}

Argyris, J. H., 1963, Recent Advances in Matrix Methods of Structural Analysis, Pergamon Press, Elmsford, New York.

Arpaci, V. S., 1966, Conduction Heat Transfer, Addison-Wesley Publishing Company.

Camprub, N., Colominas, I., Navarrina, F. and Casteleiro, M., 2000, Galerkin, Least-Squares and G.L.S. numerical approaches for convective-diffusive transport problems in engineering, European Congress on Computational Methods in Applied Sciences and Engineering.

Clough, R. W., 1960, The Finite Element Method in Plane Stress Analysis, Proceedings of $2^{\text {nd }}$ Conf. on Electronic Computation, American Society of Civil Engineers, Pittsburgh, Penn., pp. 345-378.

Dhatt, G. and Touzot, G., 1984, The Finite Element Method Displayed, John Wiley \& Sons.

Feng, J. Q., 2001, Application of Galerkin finite-element computations in studying electrohydrodynamic problems, Journal of Electrostatic, Vol. 51-52, pp. 590-596.

Howle, L. E., 1996, A comparison of the reduced Galerkin and pseudo-spectral methods for simulation of steady Rayleigh-Bénard convection, International Journal Heat Mass Transfer, Vol. 39, No. 12, pp. 2401-2407.

Jiang, B., 1998, The Least-Squares Finite Element Method. Theory and Applications in Computational Fluid Dynamics and Electromagnetics, Springer.

Reddy, J. N., 1993, An Introduction to the Finite Element Method, Second Edition, McGraw-Hill.

Romão, E. C., 2004, Variantes do Método dos Elementos Finitos para Solução de Fenômenos Convectivo-Difusivos Bidimensional, Dissertação de Mestrado, UNESP-FEIS. (in portuguese)

Romão, E. C., Moura, L. F. M., and Silva, J. B. C., 2008. Analysis of Error in the Solution of the 2-D Diffusion Equation by Finite Element Methods. TEMA. Tendências em Matemática Aplicada e Computacional, Vol. 9, No. 2, pp. 287-298

Turner, M. J., Clough, R. W., Martin, H. C., and Topp, L. P., 1956, Stiffness and Deflection Analysis of Complex Structures, J. Aeron. Sci., Vol. 23, No. 9, pp. 805-823. 